\title{
Cobalt Chloride Induces Necroptosis in Human Colon Cancer HT-29 Cells
}

\author{
Hai-Yu Wang, Bo Zhang*
}

\begin{abstract}
Necroptosis, also known as "programmed necrosis", has emerged as a critical factor in a variety of pathological and physiological processes and is considered a cell type-specific tightly regulated process with mechanisms that may vary rather greatly due to the change of cell line. Here we used HT-29, a human colon cancer cell line, to establish a necroptosis model and elucidate associated mechanisms. We discovered that cobalt chloride, a reagent that could induce hypoxia-inducible factor-1 $\alpha($ HIF1 $\alpha)$ expression and therefore mimic the hypoxic microenvironment of tumor tissue in some aspects induces necroptosis in HT-29 cells when caspase activity is compromised. On the other hand, apoptosis appears to be the predominant death form when caspases are functioning normally. HT-29 cells demonstrated significantly increased RIPK1, RIPK3 and MLKL expression in response to cobalt chloride plus z-VAD treatment, which was accompanied by drastically increased IL1 $\alpha$ and IL6 expression, substantiating the notion that necrosis can induce profound immune reactions. The RIPK1 kinase inhibitor necrostatin-1 and the ROS scavenger NAC each could prevent necrosis in HT-29 cells and the efficiency was enhanced by combined treatment. Thus by building up a necroptosis model in human colon cancer cells, we uncovered that mechanically RIP kinases collaborate with ROS during necrosis promoted by cobalt chloride plus z-VAD, which leads to inflammation. Necroptosis may present a new target for therapeutic intervention in cancer cells that are resistant to apoptotic cell death.
\end{abstract}

Keywords: Cobalt chloride - colon cancer cells - necroptosis - RIPK1 - ROS

Asian Pac J Cancer Prev, 16 (6), 2569-2574

\section{Introduction}

Colon cancer is the third most common malignant disease in men and the second in women worldwide and it is seen with substantially increasing mortality in the Asia-Pacific region (Ku et al., 2012). Besides surgical procedures, adjuvant chemotherapy only benefit highrisk stage IIandIII colon cancer patients slightly with approximately 20 months median overall survival (Ku et al., 2012). Considering its high financial cost and toxic effects, it is critical to discover potent targeted treatment to fight colon cancer.

Understanding cell death mechanisms in cancer cells and how the immune system responds to it is pivotal in unraveling effective cancer treatment. Apoptosis and necrosis are two major forms of cell death (Wang et al., 2008; ME, 2011; Sun et al., 2012). Apoptosis is executed by intracellular cysteine protease family of caspases. Conversely, necrosis has been perceived as a passive and unregulated cellular demise resulted from unmanageable physical damages ( Wang et al., 2008; ME, 2011; Sun et al., 2012). However, accumulating evidence indicates that necrosis is involved in a variety of physiological processes including T lymphocyte development, bacterial and viral infection, neurodegenerative diseases, auto-immune disorders, as well as ischemia-reperfusion injury, which underscores the crucial role of programmed necrosis in maintaining metazoans stable (Cho et al., 2009; Welz et al., 2011; Zhang et al., 2011; Narayan et al., 2012; Saddoughi et al., 2013). Furthermore some necrosis can be induced by regulated signal transduction pathways such as receptor interacting protein (RIP) kinase. Researches showed that in certain cells deficient for caspase- 8 or treated with the pan-caspase inhibitor $\mathrm{z}$-Vad, stimulation of death domain receptors such as the Fas and TNFR family causes a RIP1 kinase-dependent programmed necrotic cell death instead of apoptosis ( Wang et al., 2008; ME, 2011; Sun et al., 2012). Other studies have suggest otherwise (Fu et al., 2013). Programmed necrotic cell death induced by either alkylating or oxidizing agents has been shown to be independent of the RIP kinase-dependent necroptotic pathway (Fu et al., 2013).

Cobalt chloride is among the most well-established hypoxia mimics in the generation of ROS and activation of HIF1 $\alpha$, p53, p21 and PCNA, which is widely used in a variety of tissues and cell lines to create anemia and hypoxia environment (Zou, 2001). Here we used cobalt chloride to mimic the hypoxia microenvironment of 
colonic neoplasm thus studying necroptosis in a more physiological context.

By establishing a human colon cancer model of necroptosis with cobalt chloride, we have shown that in the presence of pan-caspase inhibitor $\mathrm{z}$-Vad, necrosis becomes the predominant form of tumor cell loss. Using a combination of morphological, biochemical, genetic, and pharmacological investigations, we further identified RIP kinases and ROS as essential mediators during this sophisticated process.

\section{Materials and Methods}

\section{Cell culture}

The human colon cancer cell line HT-29 was obtained from American Type Culture Collection (Manassas, VA). Cells were grown in Maccoy's 5A medium supplemented with $10 \%$ fetal bovine serum (FBS, Hyclone Laboratories Inc., Logan, UT) and penicillin (100U/ml) and streptomycin $(100 \mathrm{mg} / \mathrm{ml})$.Cultures were maintained at $37^{\circ} \mathrm{C}$ in a humidified atmosphere of $5 \% \mathrm{CO} 2$.

\section{Antibodies and reagents}

TNF- $\alpha, \mathrm{z}$-VAD were obtained from R\&D systems (R\&D,MN). Necrostatin-1 was purchased from Santa Cruz Biotechnology (Santa Cruz, CA).Propidium iodide and DCFHDA were from Sigma-Aldridge (SigmaAldridge, MO). Cobalt Chloride was obtained from SCR (SCR, shanghai).Rip1 antibody was obtained from BD Biosciences. Anti-rabbit IgG-HRP and GDPDH antibody were purchased form Sangon (Sangon, shanghai). Antimouse IgG-HRP was purchased from Daco (Glostrup, Denmark).

\section{Fluorescence microscropy}

For detection of necrosis and apoptosis, $1.5 \times 104$ cells were seeded per well in a 24 -well plate. Following 12 hours incubation for adherence, cells were stimulated as previously described for $48 \mathrm{hrs}$. Subsequently, cells were incubated with propidium iodide $(50 \mu \mathrm{g} / \mathrm{ml}$,) and annexin V-HITC $(5 \mu \mathrm{l}, \mathrm{BD}$ Biosciences) for $15 \mathrm{~min}$ at room temperature immediately followed by fluorescence microscopy. Digital images were processed in an identical manner using the advanced DP controller software.

\section{Quantitative real-time PCR analysis}

Total RNA was extracted from cultured cells using Trizol reagent (Invitrogen, Carlsbad, California, USA) and dissolved in diethylpyrocarbonate treated (DEPC) water. cDNA was synthesized using the Takara Reverse Transcription System Kit (Takara Biotechnology Co. Ltd., Japan) according to the manufacturer's instruction. Real time quantitative RT-PCR was performed using the Sybr green premix kit (BioRad). TPT-1 was used as a housekeeping gene. The following primers are used in this assay:RIPK 1 :sense TGGGCGTCATCATAGAGGAAG, and anti-sense CGCCTTTTCCATGTAAGTAGCA; RIPK3: sense AATTCGTGCTGCGCCTAGAAG, and anti-sense TCGTGCAGGTAAAACATCCCA; MLKL: sense AAGCTTGCAGGATTTGAGTTGA, and anti-sense CAGAGGACGATTCCAAAGCTGT;IL-
$1 \alpha$ :sense AGATGCCTGAGATACCCAAAACC, and anti-sense CCAAGCACACCCAGTAGTCT;IL-6:sense ACTCACCTCTTCAGAACGAATTG, and anti-sense CCATCTTTGGAAGgTTCAGGTTG. The PCR conditions were as following: $95^{\circ} \mathrm{Cfor} 3 \mathrm{~min}$ followed by 40 cycles at $95^{\circ} \mathrm{C}$ for $10 \mathrm{~s}$ and $55^{\circ} \mathrm{Cfor} 30 \mathrm{~s}$. All reactions were performed in triplicate both biologically and technically.

\section{Flow cytometry analysis}

Enumeration of apoptotic cells was done by using FITC conjugated Annexin V and PI (BD Pharmingen, San Jose, CA). Cells were washed twice in cold PBS and resuspended in Annexin V-binding buffer (BD Pharmingen) at a concentration of $1 \times 10^{6}$ per $\mathrm{ml}$. This suspension $(100 \mu \mathrm{l})$ was stained with $5 \mu \mathrm{l}$ of Annexin $\mathrm{V}$-FITC and $5 \mu \mathrm{I}$ PI. These cells were gently vortexed and incubated for $15 \mathrm{~min}$ at room temperature in the dark. After addition of $400 \mu \mathrm{l}$ of binding buffer to each tube, cells were analyzed by flow cytometry.

\section{ROS measurement}

The reactive oxygen species (ROS) assay was performed according to the manufacturer's instructions. Briefly, HT-29 cells were seeded in 96-well plates at a density of $2.5 \times 10^{4}$ cells per well and incubated at $37^{\circ} \mathrm{C}$ overnight for attachment. Change the culture medium to stimuli and incubated for an hour. Cells were then washed with PBS twice followed by the addition of $100 \mu$ l of fresh culture medium without phenol red or FBS containing $25 \mu \mathrm{M}$ DCFHDA (Sigma) dissolved in dimethylsulfoxide (DMSO). After the cells were incubated for $30 \mathrm{~min}$, they were washed with PBS twice and stayed in medium without phenol red and FBS. ROS absorbances were measured at $525 \mathrm{~nm}$ using a fluorescence microplate reader.

\section{Western blotting}

Equal amounts of protein were separated by $10 \%$ SDS polyacrylamide gel electrophoresis and transferred to PVDF membranes. After blocking with 5\% non-fat milk in TBST for $1 \mathrm{~h}$, membranes were incubated overnight at $4^{\circ} \mathrm{C}$ with Rip1, GAPDH primary antibodies followed by incubation with HRP-conjugated secondary antibodies. Immunoreactive bands were detected with enhanced chemiluminescent HRP substrate (Millipore, Bollerica, MA).

\section{Statistical analysis}

Data are presented as the mean \pm standard error of the mean (SEM). Values were compared and analyzed using Student's t-test. We considered P-values less than 0.05 to be statistically significant.

\section{Results}

Cobalt chloride induces necrosis in HT-29 cells

It has been established that death-inducing cytokines as TNF- $\alpha$ causes necrosis in HT-29 cell line when caspases are inhibited (Wang et al., 2008; ME, 2011; Sun et al., 2012). We discovered that necrosis also occurred 
when cells were treated with cobalt chloride and even exacerbated necrosis emerged when cells were treated with cobalt chloride plus z-VAD (Figures 1A and 1B) while no major differences have been observed in apoptosis (Figure S1). We also noticed that cobalt chloride alone has greater necrosis-inducing effect than TNF- $\alpha$ (data not shown).To verify this cell death was indeed necrosis, cells treated with either cobalt chloride or cobalt chloride plus z-VAD were analyzed by transmission electron microscopy (TEM). Surprisingly, most cells treated with cobalt chloride exhibited apoptotic morphology changes as condensed chromatin and plasma membrane blebbing while a significant percentage of cells treated with cobalt chloride plus z-VAD displayed mitochondria swelling and discontinuous cytoplasmic membrane which are typical necrosis morphology (Figure 1C). We suspected that the lack of necrosis morphology in cells treated with cobalt chloride may attribute to the undistinguishable essence between late stage apoptosis and necrosis with propidium iodide staining.

\section{RIPK1-RIPK3 axis is required for necrosis induced by} cobalt chloride

Since RIPK1/RIPK3/MLKL complex is the bestcharacterized complex during necroptosis which is fundamental to cytokines as TNF- $\alpha$, TRAIL, CD95 (Wang et al., 2008; ME, 2011; Sun et al., 2012) but is dispensable to either alkylating or oxidizing agents $(\mathrm{Fu}$ et al., 2013), we checked whether it is required for the observed necrosis caused by cobalt chloride. HT-29 cells under cobalt chloride plus z-VAD treatment expressed increased level of RIPK1, RIPK3 which expression steadily augmented over time while in cells treated with cobalt chloride alone RIPK1 RIPK3 had higher expression
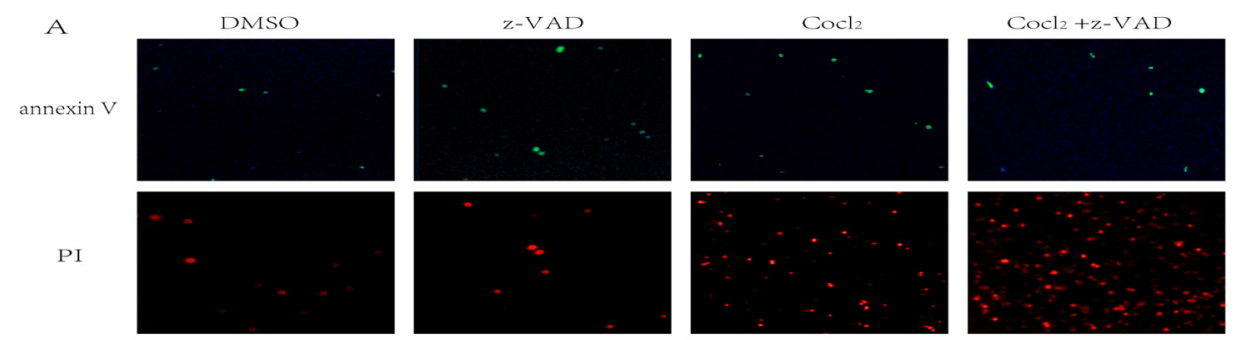

$\mathrm{B}$
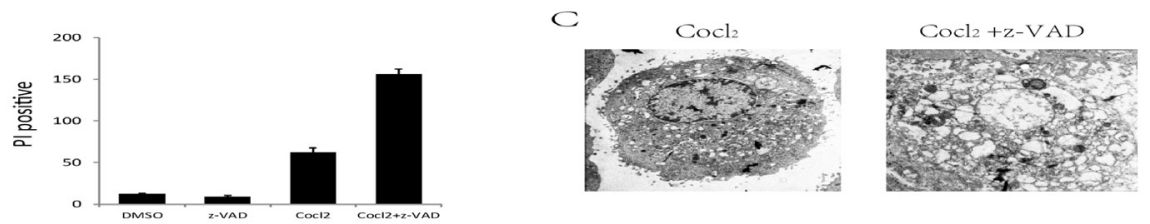

Figure 1. Cobalt Chloride Induces Necrosis in HT-29 Cells. (A and B)HT-29 colon cancer cells were treated with DMSO, $20 \mu \mathrm{M}$ z-VAD and/or $300 \mu \mathrm{M}$ cobalt chloride for $24 \mathrm{hr}$. Identical concentrations were used in later experiment unless otherwise stated. Cell viability were determined by propidium iodide(PI) and annexin V staining(A). PI positive cell number was counted(B). Data are represented as mean \pm standard deviation of different microscopic fields. (C)Electron microscopy of HT-29 cells treated for 24h with cobalt chloride or cobalt chloride with z-VAD. All experiments were at least repeated three times with similar results
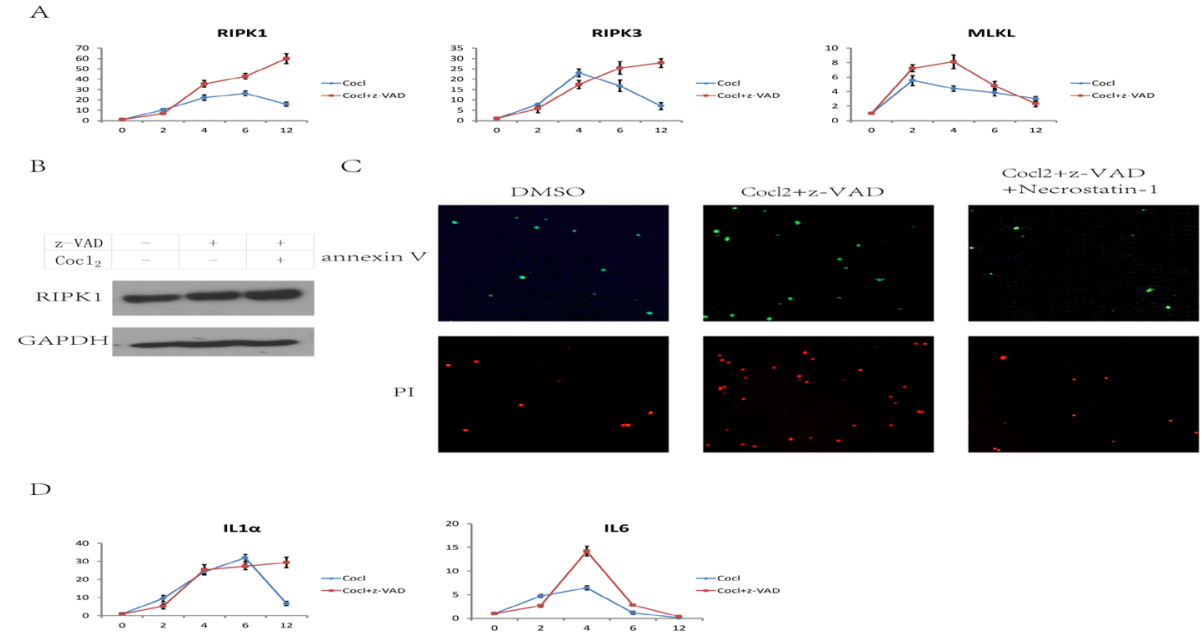

Figure 2. RIPK1-RIPK3 Axis is Required for Necrosis Induced by Cobalt Chloride. (A)HT-29 cells were treated with DMSO, cobalt chloride and/or z-VAD for indicated time. Quantitative polymerase chain reaction with reverse transcription (qRT-PCR) analysis of RIPK1, RIPK3,MLKL .All graphs show mean \pm standard deviation. P<0.05. (B)HT-29 cells were treated with DMSO, cobalt chloride and/or z-VAD for $12 \mathrm{hr}$. Cell lysates were collected and subjected to western blot analysis of RIPK1 and GAPDH levels. (C) HT-29 colon cancer cells were treated with DMSO, cobalt chloride plus z-VAD and cobalt chloride plus z-VAD plus necrostatin-1 $(40 \mu \mathrm{M})$ for $24 \mathrm{hr}$ and then stained with PI and annexin V. (D) qRT-PCR analysis of IL1 $\alpha$ and IL6 of HT29 cells with indicated treatment during indicated time. All graphs show mean \pm standard deviation. All experiments were at least repeated three times with similar results 
Hai-Yu Wang and Bo Zhang

during earlier hours and then ameliorated (Figure 2A). MLKL expression peaked at 2 hours during both treatment (Figure 2A).RIPK1 expression was further confirmed by Western blotting (Figure 2B) after 12 hours of stimulation and we ruled out the possible effects of $\mathrm{z}$-VAD alone (Figure S2 and data not shown). As a validation to our finding, necrostatin-1, an RIPK1 kinase inhibitor rendered HT-29 cells resistant to this type of cell death (Figure 2C). Furthermore necrosis is always associated with ensuing inflammation reaction (Zheng et al., 2013). In consistence with this notion, we find increasing levels of IL1- $\alpha$ and IL6 (Figure 2D) overtime. We also noticed that there was certain basal level of endogenous RIPK1 in HT-29 cells which may accordingly render HT-29 susceptible to programmed necrotic death in the first place.

ROS is indispensable for Cobalt chloride-induced necrosis

RIPK1 is a key regulator of necroptosis and its expression level has been shown to correlate with responsiveness to programmed necrosis ( Wang et al., 2008; ME, 2011; Sun et al., 2012). We monitored RIPK1 expression level during early hours of necrosis and noticed a different trend between cobalt chloride and typical TNF-atreatment indicating that there may be different mechanisms lying here (Figure 3A) meanwhile z-VAD alone didn't impose major effects (Figure S3). Since cobalt chloride alone could generate ROS (Zou W, 2001) we tested if ROS played a significant role under

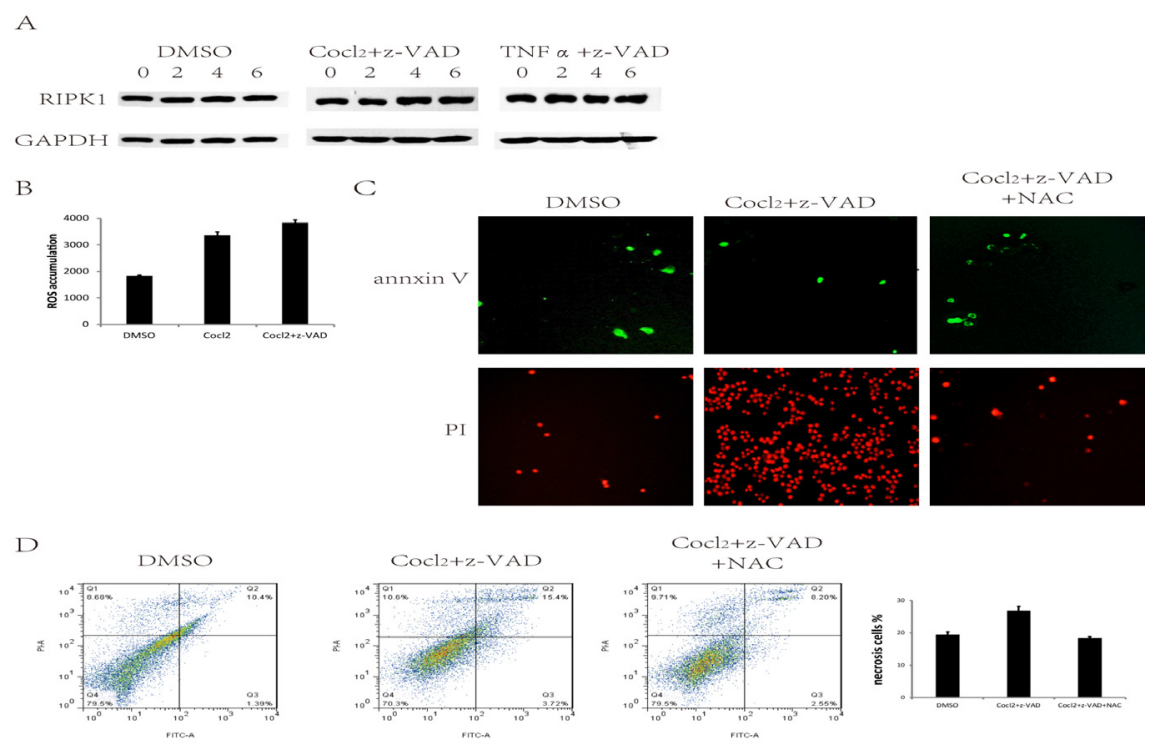

Figure 3. ROS is Indispensable for Cobalt Chloride-Induced Necrosis. (A) HT-29 cells were treated with DMSO, cobalt chloride and/or z-VAD, TNF $\alpha$ and/or z-VAD for indicated time. Cell lysates were collected and subjected to western blot analysis of RIPK1 and GAPDH levels. (B) HT-29 cells were treated with DMSO, cobalt chloride and/or z-VAD for 2hr. Cells were incubated with $25 \mu \mathrm{M}$ DCFHDA. Intracellular ROS levels were quantified with a fluorescence microplate reader. Data are mean \pm standard deviation. $\mathrm{P}<0.05$ (C and D) Representative fluorescence microscopy and FACS analysis of annexin $\mathrm{V}$ and propidium iodide(PI) staining $2 \mathrm{~h}$ after stimulation with cobalt chloride plus $\mathrm{z}$-VAD and cobalt chloride plus z-VAD plus NAC $(1 \mathrm{mM})$.All experiments were at least repeated three times with similar results

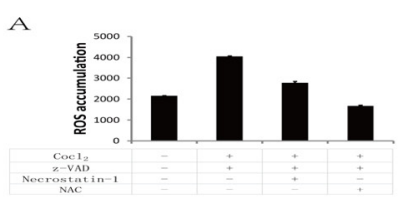

B
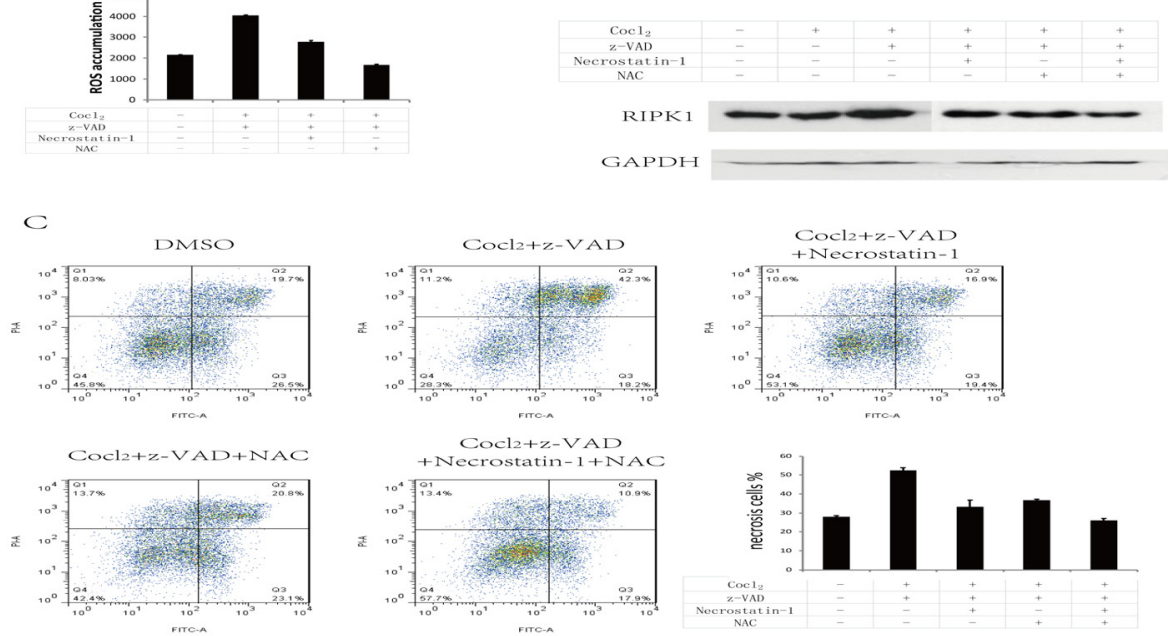

Figure 4. ROS Pathway and RIPK1-RIPK3 Axis Collaborate During Necroptosis. (A) After indicated treatment for $2 \mathrm{hr}$, cells were incubated with $25 \mu \mathrm{M}$ DCFHDA. Intracellular ROS levels were quantified with a fluorescence microplate reader. Data are mean \pm standard deviation. $\mathrm{P}<0.05$. (B) HT-29 cells were treated as indicated for $12 \mathrm{hr}$. Cell lysates were collected and subjected to western blot analysis of RIPK1 and GAPDH levels. (C) Representative FACS analysis of annexin V and propidium iodide(PI) staining $2 \mathrm{~h}$ after stimulation as indicated. All experiments were at least repeated three times with similar results 
these circumstances. Indeed, ROS level increased nearly twofold in cells treated with cobalt chloride plus z-VAD (Figure 3B) while z-VAD alone didn't increase ROS (Figure S4). PI/annexin V staining and FACS analysis further confirmed our proposition that by adding ROS scavenger NAC necrosis was potently impeded (Figure 3C and 3D). It also came to our acknowledgement that there is no noticeable differences in ROS production between cobalt chloride and cobalt chloride plus z-VAD (Figure 3B), suggesting that ROS level may not directly correlated to programmed necrosis and there are more complicated regulations involved at the crossroads of apoptosis and necroptosis.

\section{ROS pathway and RIPK1-RIPK3 axis collaborate during necroptosis}

Because RIPK1-RIPK3 signaling can promote mitochondrial ROS production (Sakurai et al., 2008), necrostatin-1 was used to eliminate the possibility that ROS required for necrosis was induced by RIPK1-RIPK3 pathway. Indeed, ROS production increase was hindered but not completely obstructed by necrostatin-1 (Figure $4 \mathrm{~A})$, implying that RIP kinase did regulate downstream ROS production but there were more pathways responsible for ROS accumulation. We next sought to determine if RIPK1-RIPK3 signaling and ROS functions cohesively. As shown in Figure 4B, RIPK1 expression was elevated in cobalt chloride plus z-VAD treated cells and was substantially inhibited with the combination of RIPK1 kinase inhibitor necrostatin-1 and anti-oxidant NAC. Consistent with this, flow cytometry analysis showed that the presence of both necrostatin- 1 and NAC significantly reduced PI positive cells (Figure 4C).Collectively these data demonstrate that ROS and RIPK1-RIPK3 cooperated during HT-29 human colon cancer cell necroptosis induced by cobalt chloride and z-VAD.

\section{Discussion}

Colon cancer is seen with increasing frequency in the Asia-Pacific region and it is one of the most important causes of cancer mortality worldwide. A great comprehension of tumor cell loss and its ensuing immune responses is important to elicit a successful anti-cancer treatment (Ullrich et al., 2008). While many of the cellular aspects of apoptosis have been characterized, the initiating factors and regulating mechanisms of programmed necrotic tumor cell death are only beginning to be found. In this study we established a programmed necrosis model in HT-29 human colon cancer cell line with cobalt chloride, a hypoxia-inducing agent mimicking tumor microenvironment. Furthermore, we found that additional pan-caspase inhibitor z-VAD treatment increases necrotic cell death during tumorigenesis but doesn't affect apoptosis (Figure 1). These findings clearly demonstrate programmed necrosis occurs during tumorigenesis which is further revealed when caspases are inhibited. We then utilized this model to investigate the essential mediators of this specific process.

RIPK1 is a serine/threonine adaptor kinase that acts downstream of downstream of death domain receptors and is essential for both cell survival and death (Green et al., 2011). Although the kinase activity of RIPK1 is not required for NF- $x$ B activation (He et al., 2009), such activity is crucial for both caspases activation and necrosis induction. Consistent with this RIPK1 kinase inhibitor necrostatin-1 cotreatment effectively suppress necrotic tumor cell death (Figure 2). RIPK1 is reported to form a pro-necrosis complex with RIPK3/FADD/caspase-8 when treated with death cytokines such as TNF $\alpha$, Fas (Oberst et al., 2011). Since necrosis is differently regulated in this model (Figure 2), the effect protein of RIPK1 under this circumstance is unknown. It should be our priority in the future to identify the unknown components of such pathway. Some study showed that DNA damage triggered by alkylating chemotherapy agents activates a RIPK1independent necrotic signaling pathway which was carried out with no full consideration of tumor heterogeneity and microenvironment (Junttila and de Sauvage, 2013). By further exploiting this model, we could look into the programmed necrotic death induced by alkylating agents in a more physiological context and thus might uncover potential therapeutic targets.

Reactive oxygen species (ROS) have been implicated in TNF- $\alpha$ induced necrosis. Nox1 NAPDH oxidase has been shown to work downstream of RIPK1 to generate ROS in L929 and MEF cells (Whelan RS, 2012). Alternatively, RIP3 activates metabolic enzymes such as glutamate dehydrogenase 1 , and thereby increases mitochondrial ROS production (Tu et al., 2009). However, ROS dependence seems cell-type specific with data showing that ROS quenching failed to prevent necrosis in HT-29 cells treated with TNF- $\alpha$, Smac mimetic and z-VAD (ME, 2011). Our data indicated that ROS was indispensable during necrosis induced by cobalt chloride plus z-VAD treated HT-29 cells (Figure 3)and only part of which that was generated by RIPK1 (Figure 4).

Since many cancer cell types can evade apoptotic cell death through deficiencies in key apoptotic proteins or by inhibition of apoptotic pathways and evasion of apoptosis is recognized to result in resistance to anti-cancer therapies (de Bruin and Medema, 2008; Krysko and Vandenabeele, 2008), elucidation of the necrotic signaling cascades will certainly enable targeted therapeutics in eliminating apoptosis-resistant cancer cells. Indeed recent studies showed that apoptosis is dispensable for complete tumor regression after alkylating agent chemotherapy because other forms of cell death can be activated (Krysko et al., 2012), including programmed necrosis. Moreover, necrosis promotes the clearance of apoptosis-deficient tumors by amplifying the initial damage signal through activating inflammatory response which destroys damaged cells and facilitates the cleaning up of cell debris (Kaczmarek et al., 2013). Cancer cells also exhibit increased ROS generation that may promote cell proliferation and, in many cases, might be coupled with redox adaptation to promote cell survival and drug resistance (Saeidnia and Abdollahi, 2013). Targeting this unique biochemical alteration might be a feasible approach to achieve therapeutic selectivity and to overcome drug resistance. In preclinical models, combination of ROS-generating agents and drugs that inhibit ROS elimination showed great potency to promote 
Hai-Yu Wang and Bo Zhang

ROS accumulation and enhance cancer cell cytotoxicity (Trachootham et al., 2009; Sosa et al., 2013). In this work, we identify that during necrosis in HT-29 cells induced by cobalt chloride plus z-VAD treatment RIPK1 kinase acts in concert with ROS. Simultaneous inhibition of both RIP1 kinase and ROS pathways with small molecule compounds provides significant protection of programmed cell death (Figure 4).

There is convincing preclinical and accumulating clinical evidence in support of the notion that successful antineoplastic therapies reinstate immunosurveillance (Motz and Coukos, 2013). Unlike apoptosis, programmed necrosis is highly inflammatory. Extracellular HMGB1 released during necroptosis generally boosts immune responses (Duprez et al., 2011). Therefore programmed necrosis presents potential targets for therapeutic intervention. However, whether the inflammation associated with necrosis is desirable or leads to further tumor growth is under debate (Krysko et al., 2012). Our study demonstrated a crucial link between necroptosis, ROS and immune response. Their complexities underscore the need to comprehend the interplay between them more profoundly. An improved knowledge of these aspects will facilitate the rational design of highly efficient, synergistic regimens for anticancer therapy.

\section{References}

Cho YS, Challa S, Moquin D, et al (2009). Phosphorylationdriven assembly of the RIP1-RIP3 complex regulates programmed necrosis and virus-induced inflammation. Cell, 137, 1112-23.

de Bruin EC, Medema JP (2008). Apoptosis and non-apoptotic deaths in cancer development and treatment response. Cancer Treat Rev, 34, 737-49.

Duprez L, Takahashi N, Van Hauwermeiren F, et al (2011). RIP kinase-dependent necrosis drives lethal systemic inflammatory response syndrome. Immunity, 35, 908-18.

Fu D, Jordan JJ, Samson LD (2013). Human ALKBH7 is required for alkylation and oxidation-induced programmed necrosis. Genes Dev, 27, 1089-1100.

Green DR, Oberst A, Dillon CP, et al (2011). RIPK-dependent necrosis and its regulation by caspases: a mystery in five acts. Mol Cell, 44, 9-16.

He S, Wang L, Miao L, et al (2009). Receptor interacting protein kinase- 3 determines cellular necrotic response to TNF-alpha. Cell, 137, 1100-11.

Junttila MR, de Sauvage FJ (2013). Influence of tumour microenvironment heterogeneity on therapeutic response. Nature, 501, 346-54.

Kaczmarek A, Vandenabeele P, Krysko DV (2013). Necroptosis: the release of damage-associated molecular patterns and its physiological relevance. Immunity, 38, 209-223.

Krysko DV, Garg AD, Kaczmarek A, et al (2012). Immunogenic cell death and DAMPs in cancer therapy. Nat Rev Cancer, 12, 860-75.

Krysko DV, Vandenabeele P (2008). From regulation of dying cell engulfment to development of anti-cancer therapy. Cell Death Differ, 15, 29-38.

$\mathrm{Ku} \mathrm{G}$, Tan IB, Yau T, et al (2012). Management of colon cancer: resource-stratified guidelines from the Asian Oncology Summit 2012. Lancet Oncol, 13, 470-481.

ME P. (2011). Programmed cell death: Apoptosis meets necrosis. Nature, 471, 310-2.
Motz GT, Coukos G (2013). Deciphering and reversing tumor immune suppression. Immunity, 39, 61-73.

Narayan N, Lee IH, Borenstein R, et al (2012). The NADdependent deacetylase SIRT2 is required for programmed necrosis. Nature, 492, 199-204.

Oberst A, Dillon CP, Weinlich R, et al (2011). Catalytic activity of the caspase-8-FLIP (L) complex inhibits RIPK3dependent necrosis. Nature, 471, 363-7.

Saddoughi SA, Gencer S, Peterson YK, et al (2013). Sphingosine analogue drug FTY720 targets I2PP2A/SET and mediates lung tumour suppression via activation of PP2A-RIPK1dependent necroptosis. EMBO Mol Med, 5, 105-121.

Saeidnia S, Abdollahi M (2013). Antioxidants: friends or foe in prevention or treatment of cancer: the debate of the century. Toxicol Appl Pharm, 271, 49-63.

Sakurai T, He G, Matsuzawa A, et al (2008). Hepatocyte necrosis induced by oxidative stress and IL-1 alpha release mediate carcinogen-induced compensatory proliferation and liver tumorigenesis. Cancer Cell, 14, 156-65.

Sosa V, Moline T, Somoza R, et al (2013). Oxidative stress and cancer: an overview. Ageing Res Rev, 12, 376-90.

Sun L, Wang H, Wang Z, et al (2012). Mixed lineage kinase domain-like protein mediates necrosis signaling downstream of RIP3 kinase. Cell, 148, 213-27.

Trachootham D, Alexandre J, Huang P (2009). Targeting cancer cells by ROS-mediated mechanisms: a radical therapeutic approach? Nat Rev Drug Discov, 8, 579-91.

Tu HC, Ren D, Wang GX, et al (2009). The p53-cathepsin axis cooperates with ROS to activate programmed necrotic death upon DNA damage. Proc Natl Acad Sci USA, 106, 1093-8.

Ullrich E, Bonmort M, Mignot G, et al (2008). Tumor stress, cell death and the ensuing immune response. Cell Death Differ, 15, 21-8.

Wang L, Du F, Wang X (2008). TNF-alpha induces two distinct caspase-8 activation pathways. Cell, 133, 693-703.

Welz PS, Wullaert A, Vlantis K, et al (2011). FADD prevents RIP3-mediated epithelial cell necrosis and chronic intestinal inflammation. Nature, 477, 330-4.

Whelan RS KK, Wei AC, Chen Y, et al (2012). Bax regulates primary necrosis through mitochondrial dynamics. Proc Natl Acad Sci USA, 109, 6566-71.

Zhang H, Zhou X, McQuade T, et al (2011). Functional complementation between FADD and RIP1 in embryos and lymphocytes. Nature, 471, 373-6.

Zheng Y, Humphry M, Maguire JJ, et al (2013). Intracellular interleukin-1 receptor 2 binding prevents cleavage and activity of interleukin-1alpha, controlling necrosis-induced sterile inflammation. Immunity, 38, 285-95.

Zou W, Xu W, Huo H, et al (2001). Cobalt chloride induces $\mathrm{PC} 12$ cells apoptosis through reactive oxygen species and accompanied by AP-1 activation. J Neurosci Res, 64, 646-53. 